\title{
Bionic intrafascicular interfaces for recording and stimulating peripheral nerve fibers
}

\author{
Ranu Jung ${ }^{*}, \uparrow, 1$, James J Abbas ${ }^{\dagger, 2}$, Sathyakumar Kuntaegowdanahalli ${ }^{1}$ \& Anil K Thota ${ }^{1}$ \\ ${ }^{1}$ Department of Biomedical Engineering, Florida International University, EC2602, 10555 W Flagler Street, Miami, FL 33134, USA \\ ${ }^{2}$ Center for Adaptive Neural Systems, School of Biological \& Health Systems Engineering, PO Box 879709 Arizona State University, \\ Tempe, AZ 85287-9709, USA \\ * Author for correspondence: rjung@fiu.edu \\ ${ }^{\dagger}$ Authors contributed equally
}

The network of peripheral nerves presents extraordinary potential for modulating and/or monitoring the functioning of internal organs or the brain. The degree to which these pathways can be used to influence or observe neural activity patterns will depend greatly on the quality and specificity of the bionic interface. The anatomical organization, which consists of multiple nerve fibers clustered into fascicles within a nerve bundle, presents opportunities and challenges that may necessitate insertion of electrodes into individual fascicles to achieve the specificity that may be required for many clinical applications. This manuscript reviews the current state-of-the-art in bionic intrafascicular interfaces, presents specific concerns for stimulation and recording, describes key implementation considerations and discusses challenges for future designs of bionic intrafascicular interfaces.

First draft submitted: 2 October 2017; Accepted for publication: 13 November 2017; Published online: 14 December 2017

Keywords: autonomic nervous system interface $\bullet$ biocompatibility $\bullet$ fascicle $\bullet$ intrafascicular electrode $\bullet$ neural recording • neural stimulation $\bullet$ peripheral nerve $\bullet$ peripheral nerve interface

The peripheral nervous system offers unprecedented access for neuromodulation of the central nervous system (CNS) or end organs. The nervous system functions by generating patterns of neural activity, which underlie sensation and perception as well as control of movement, cardiovascular, endocrine, immune and other systems. To influence neural activity for desired outcomes, neural interface technology must access the appropriate peripheral nerve tissue, activate it in a focal-targeted manner and alter the pattern formation mechanisms [1]. Mammalian peripheral nerves generally consist of several fascicles that have numerous myelinated and unmyelinated nerve fibers, include a complex network of blood vessels and have several layers of connective tissue [2]. Hence, they present considerable challenges and design constraints to the development of neural interfaces for recording activity of specific fibers and/or selectively stimulating them.

Currently, the most widely used approach to interfacing with peripheral nerve fibers is to position an electrode, or an array of electrodes, using a cuff-like structure that wraps around the nerve [3-7]. This extraneural approach seeks to avoid disruption or damage to the anatomical structures within the nerve while utilizing a comparatively simple implantation procedure. However, the fascicular structure of the nerve and the insulating properties of its connective tissue impair the ability of the electrodes to selectively stimulate or record from individual or small populations of fibers. This shortcoming may be critical for applications that require localized activation or recording from small groups of fibers with high specificity [2].

A case in point is the use of an extraneural cuff to activate the vagus nerve. Despite showing promising signs in the treatment for epilepsy and rheumatoid arthritis, vagus nerve stimulation treatment using extraneural cuff electrodes is plagued by several side effects, and very modest success has been shown in recent applications for treatment of depression [7-11]. One of the main reasons is our current inability to produce natural patterns of activation, which may require localized stimulation across many channels in an asynchronous manner. The vagus nerve has $>100,000$ nerve fibers, about $80 \%$ of which are unmyelinated afferent fibers linking the thoracic and abdominal viscera and the CNS [12]. Of the remaining nerve fibers, $15 \%$ are large myelinated fibers and most of the 
remaining 5\% are small myelinated efferent fibers innervating the thoracic viscera [12]. Techniques that have been used for extraneural electrical stimulation of the vagus nerve preferentially activate large nerve fibers and the cuff offers little control over the distribution of stimulation intensity within the nerve. Given the large diversity of fibers distributed throughout the vagus nerve, it is clear that there is a possibility of unintended effects of stimulation. In other applications of peripheral nerve stimulation, advances in the design of cuff electrodes have attempted to overcome the problem of limited selectivity of specific fascicles by reshaping the nerve to increase its perimeter and position fascicles near electrode contacts $[4,13]$, by using multiple electrodes around the nerve to increase the likelihood that a targeted structure is near a contact and to facilitate current steering [14] and by selecting specific electrodes to shape the electric field [4,13-15]. However, these electrodes still have limited selectivity for stimulating or recording specific sites within a fascicle and it appears unlikely that these advances in electrode design will be sufficient to eliminate side effects such as those observed with vagus nerve stimulation.

Neural interface systems based on intrafascicular electrodes may address these limitations of the extraneural approach. Intrafascicular electrodes offer access to the microstructures and functional microdomains of peripheral nerves thereby allowing targeted stimulation to alter patterned output for specific end-organ control or for controlling activation in specific pathways to the brain $[2,16]$. The additional ability to record and decode neural activity from specific neural fibers could considerably accelerate the development of advanced closed-loop control systems.

\section{Intrafascicular electrodes: stimulation}

Peripheral nerves carry bundles of afferent and efferent axonal nerve fibers that connect sensors and effectors to the CNS [2]. The nerve fibers are separated from each other by a collagenous matrix, called endoneurium, to form fascicles, which are encapsulated by a cellular perineurium. A nerve can have one or more fascicles, which are embedded in a collagenous matrix and encapsulated by the epineurium. Fascicles can fuse along the length of the nerve but still maintain some somatotopic organization [17-20] and fibers within a fascicle may also exhibit functional organization [2,21-23]. The axonal fibers themselves can be myelinated or unmyelinated and, depending on their diameter, have different thresholds for electrical stimulation and conduction velocities.

Several electrode technologies have been developed to establish a chronic bionic neural interface. The choice of the electrode depends on many factors including safety, biocompatibility, long-term stability, ease of implantation, mechanical characteristics, electrochemical characteristics and economics.

Intraneural electrodes are designed to penetrate the epineurium to lie within a nerve, with the electrode recording/stimulation site either outside of and between fascicles or inside a fascicle itself [24,25]. The perineurium around the fascicles plays a critical role in impacting the ability to chronically record or stimulate targeted sets of nerve fibers [2]. The perineurium confines the current flow primarily within a fascicle thereby limiting the ability of an extrafascicular electrode to record individual axonal discharge or population activity from functionally discrete populations of nerve fibers [2]. In addition, the current flows easily axially in the nerve than across the nerve because of the longitudinal arrangement of myelin in the fascicle [26]. Similarly, it limits the ability of extrafascicular electrical stimulation to recruit specific nerve fibers within the fascicle, preferentially leading to the recruitment of larger fibers [27-30]. Thus, anatomical and physiological constraints dictate that an effective peripheral nerve interface for providing discrete distally referred sensations, for recruiting fibers based on size and distance from the electrode, or for recording specific command signals from the CNS would benefit from placing electrode sites within the perineurium of individual nerve fascicles [2,31-34].

This intrafascicular placement of the neural interface can be accomplished by using punctate penetrating arrays such as the Utah Slanted Electrode Array (USEA) [31-37], the transverse intrafascicular multichannel electrode array (TIME) [34,38], individual longitudinal intrafascicular electrodes (LIFEs) [32] or thin-film LIFEs [33,39]. Chronically implanted intrafascicular electrodes can elicit discrete activation of nerve fibers on electrical stimulation, as illustrated by reproducible and localized finger sensations or object discrimination capabilities in amputees [35,37-40].

The USEA, which is a variant of an original design to record and stimulate cortical tissue, consists of 100 electrodes approximately $1 \mathrm{~mm}$ in length that are arranged in a $10 \times 10$ array with electrodes spaced at $400 \mu \mathrm{m}$ and have been implanted in cat sciatic nerves for long-term evaluation [41,42]. USEAs have also been implanted in human amputees for up to 1 month [35]. These and even higher density version [43] micro electrode arrays allow access to a large population of fibers in the nerve and allow recording from multiple electrodes of an array. They are implanted perpendicular to the nerve fibers and, since the slanted array has electrodes of different lengths, they penetrate to different depths. However, while some electrode tips may penetrate a fascicle, others may land between fascicles, and the implantation procedure also does not allow for the specific alignment of electrodes with specific 
fascicles. The delivery method and the rigid structure also contribute to an enhanced risk of nerve damage. In addition, the implanted array has to be securely anchored to the nerve to minimize relative movement of the nerve fibers and the electrode contacts. USEA's with a flexible platform and a flexible interconnection cable have been designed and they have been shown to successfully record neural activity from the canine sciatic nerve chronically over 4 weeks; however, histopathological issues remained [44].

LIFE electrodes are implanted parallel to the nerve fibers. A sufficiently long length of the fascicle to accommodate the LIFE electrode is necessary. The somatotopic organization of peripheral nerves at both the fascicular and subfascicular levels allows highly selective functional and topographical access for stimulation or recording [31,33,40,45-48]. The small diameter of LIFE electrodes, which are made of platinum/iridium (Pt/Ir), and their longitudinal orientation provide a safe and stable interface to the nerve subpopulations within a nerve fascicle $[31,49]$. The small diameter, which is comparable to that of the large myelinated nerve fibers, and their flexibility provide a high degree of mechanical compatibility for the metal wires with the nerve fibers. The longitudinal orientation, which aligns the fine wire with the nerve fibers, further reduces the potential for mechanical stress-induced damage or movement. It is possible to place multiple LIFEs in one fascicle, in different fascicles within one nerve or in multiple nerves to provide access to multiple sites that are distributed in a region of the body [50]. The length of the exposed region, which is designed to be approximately equivalent to internodal spacing $(1 \mathrm{~mm})$, increases the likelihood of proximity to a node; the longitudinal placement, which aligns the exposed region parallel to the fibers, minimizes the number of fibers in close proximity. Collectively these endow the electrode with high specificity and low thresholds for activation [51]. Their geometry can also allow very low levels of charge delivery for selective activation of fibers within the fascicle. The need for low charge delivery is also a major advantage for the design of implantable electronics. By coordinating stimulation pulses delivered to LIFEs, specific patterns of activity across small groups of fibers within a given fascicle or group of fascicles could be produced.

Although LIFEs have demonstrated high selectivity and stability, for applications that require many channels of communication, the need to place many individual wires may be prohibitive. The number of LIFE electrodes that can be inserted within a given fascicle is limited by concerns about the overall volume of material and the potential for fascicular damage during multiple insertions. Several groups have designed multicontact intrafascicular probes in order to simplify deployment and increase channel counts. The thin-film longitudinal intrafascicular electrode (tfLIFE) is a microfabricated multichannel electrode on a thin polyimide base. Patterning of platinum metal on the polyimide base forms active electrodes, and the typical electrode contact size is approximately $100 \times 40 \mu \mathrm{m}$. An $80 \mu \mathrm{m}$ diameter guiding needle attached to the polyimide base is used to implant the tfLIFE such that it is approximately aligned with the nerve fibers [52]. The TIME also has multiple independent electrode contacts and is implanted perpendicular to the nerve fibers with intra- or inter-fascicular placement [33]. Under visual guidance they can be woven through specific fascicles. The biocompatibility of the TIME has been established in a chronic implantation study where TIME were implanted in the sciatic nerves of the rats for 3 months [53]. Human and animal studies show that TIME can be used for stimulation and recording from peripheral nerves $[33,34,37,38]$. Polymeric substrates to make the multielectrode arrays biomechanically and structurally compatible with peripheral nerves have been considered [54], including the design of a self-opening neural interface [55].

While the intrafascicular approach provides opportunities for high selectivity, it also has some inherent limitations. Since the electrodes must be placed within a fascicle, insertion requires a more detailed and lengthy surgical procedure [50,56]. Both the insertion procedure and long-term presence within the fascicle present risks of trauma to the nerve fibers and neighboring tissue [53]. Since highly localized access to small groups of fibers is dependent on co-localization of the electrode contact and the fibers, movement of the electrode contact relative to the target neural tissue could drastically alter the response properties $[31,45]$. Hence, use of the intrafascicular approach may be contraindicated when the anatomical target is difficult to access or subject to very high mechanical strain. While these limitations of the various intrafascicular electrodes have been addressed for some nerve targets, the advantages and disadvantages of the various intrafascicular and extrafascicular approaches should be assessed for any given application.

\section{Intrafascicular electrodes: choosing stimulation parameters}

A major advantage of intrafascicular placement of electrodes is the proximity of the nerve fibers, which can allow low charge delivery for activating the nerve fibers and greater control of the spatial spread of the electric field. The choice of the material with which the electrode is made also alters the capacity for charge delivery. Additionally, it is necessary to select stimulation parameters that will avoid tissue damage and electrode degradation. 
Cogan [57] defines macro electrodes as those that are characterized by high-charge/phase thresholds and lowcharge density thresholds. Micro electrodes on the other hand have the opposite pattern. The general boundaries for classifying neural stimulation electrodes are based on geometric surface area (GSA), with electrodes having GSA $\geq 0.001 \mathrm{~cm}^{2}$ classified as 'macro' and electrodes having GSA $\leq 0.0001 \mathrm{~cm}^{2}$ classified as 'micro'.

The USEA with small electrode tips $\left(0.00005 \mathrm{~cm}^{2}\right)$ or TIME electrodes with similarly sized electrode contacts of approximately $0.00005 \mathrm{~cm}^{2}$, are both classified as micro electrodes. On the other hand, LIFE electrodes that are made of Pt-Ir wires of approximately $25 \mu \mathrm{m}$ diameter have an active stimulation site that is $1 \mathrm{~mm}$ long and a GSA of approximately $0.0007 \mathrm{~cm}^{2}$, LIFEs are between the boundaries defined by Cogan [57], but close to the boundary for classification as a 'macro' electrode. Another study by Cogan et al. [58] in which they show that the micro electrode effects are only seen on use of electrodes with an area $<0.0002 \mathrm{~cm}^{2}$ leads to the same classification for LIFEs. Hence, the safety criterion defined for other Pt-based macro electrodes can be used as a starting point to estimate safety of chosen stimulation parameters. In particular, one widely used criterion is the Shannon criterion [59], which is derived from an empirical model based on the data collected by McCreery et al. [60]. The model postulates that tissue damage due to electrical stimulation is a function of both charge density and charge per phase. The model also provides a criterion to estimate the damage threshold based on these two parameters.

There is limited data regarding safe levels of stimulation in peripheral nerves implanted with Pt-based macro electrodes. Although the peripheral nerve environment is different from that of the cortex, the safety criterion defined for Pt-based macro electrodes in cortical tissue can be used as a starting point to estimate the safety of chosen stimulation parameters for intrafascicular electrodes. Figure 1A summarizes the data on neural injury from various studies in animal models using Pt macro electrodes. Specifically, the plot illustrates the relationship between charge density and charge per phase with histologically detectable neural injury. A conservative damage threshold line (Shannon criterion, $\mathrm{k}=1.7$ ) is extrapolated to cover all data points in the plot. Briefly, McCreery et al. implanted Pt electrodes in the parietal cortex of cats to study stimulation-induced damage [60,61]. Yuen et al. conducted studies to examine neural damage due to stimulation for up to $50 \mathrm{~h}$ using Pt disc electrodes implanted in parietal cortex of cats [62]. Pudenz et al. implanted Pt discs in cerebral cortex of cats to investigate the cause of neural damage due to electrical stimulation for $36 \mathrm{~h}$ [63]. Bullara et al. implanted an array of Pt-Ir (70-30\%) micro electrodes in the cerebral cortex of cats and showed that neurons can be activated without damage for $24 \mathrm{~h}$ at charge densities ranging from 150 to $300 \mu \mathrm{C} / \mathrm{cm}^{2}$ [64]. Agnew et al. used Pt electrodes implanted in the feline cortex to study effects of stimulation for up to $4 \mathrm{~h}$ or prolonged stimulation for $24 \mathrm{~h}$ or $23 \mathrm{~h} /$ day for 7 days [65,66]. Brown $e t$ al. studied tissue reactions due to stimulation with platinum electrode arrays implanted in cerebellums of monkeys for up to 18 days [67]. Collectively, these data provide guidelines for choosing safe levels of stimulation.

Figure 1B contains a similar plot but with data from Pt macro electrodes used in human clinical applications related to deep-brain stimulation (DBS) [60,61,63], cortical surface stimulation [68,69] and spinal cord stimulation [70]. A typical DBS electrode from Medtronic consists of an electrode lead with multiple Pt-Ir contacts with area $=0.06 \mathrm{~cm}^{2}$. Multiple human postmortem studies have indicated little tissue damage associated with chronic DBS when the stimulus parameters are within $30 \mu \mathrm{C} / \mathrm{cm}^{2}$ [71]. In their postmortem analysis of a subject who was treated with bilateral subthalamic nucleus (STN) DBS, Henderson et al. found mild cell loss, gliosis and vacuolation on one of the contacts [68]. Haberler et al. found mild gliosis in eight subjects with STN DBS [69]. Burbaud et al. reported data from a patient with chorea-acanthocytosis who was treated with DBS for 2 years. On postmortem analysis, they also concluded that DBS has little impact on the surrounding tissue [70]. It should be noted that while DBS typically employs continuous $(24 \mathrm{~h} /$ day) stimulation at high pulse rates, some of applications of peripheral nerve bionic interfaces would utilize intermittent stimulation.

The information above and in Figure 1 provides guidelines for the choice of stimulation parameters to be used with a given electrode and can motivate the development of strategies to increase the effective surface area. Various materials have been used to fabricate or coat micro electrodes in order to increase their effective surface area in order to enhance their capacity for safe charge delivery [72,73].

Figure 2 contains a plot of the charge and charge density values for the stimulus parameters tested with LIFEs in several clinical and nonclinical studies. The figure also includes the electrochemical safe limit for platinum electrodes and demarcation between regions considered safe and nonsafe based on the Shannon criteria $(\mathrm{k}=1.7)$, and a line indicating the charge density and charge per phase values for a LIFE with an area of $0.00072 \mathrm{~cm}^{2}$. Briefly, in nonclinical acute studies (open symbols in Figure 2) Nannini et al. implanted Teflon-coated $25 \mu \mathrm{m}$ Pt-Ir wire LIFEs in nerves innervating gastrocnemius and soleus muscles in cats to study muscle recruitment [74]. Yoshida et al. used similar LIFEs to demonstrate selectivity and fatigue resistance (using interleaved stimulation) of intrafascicular 


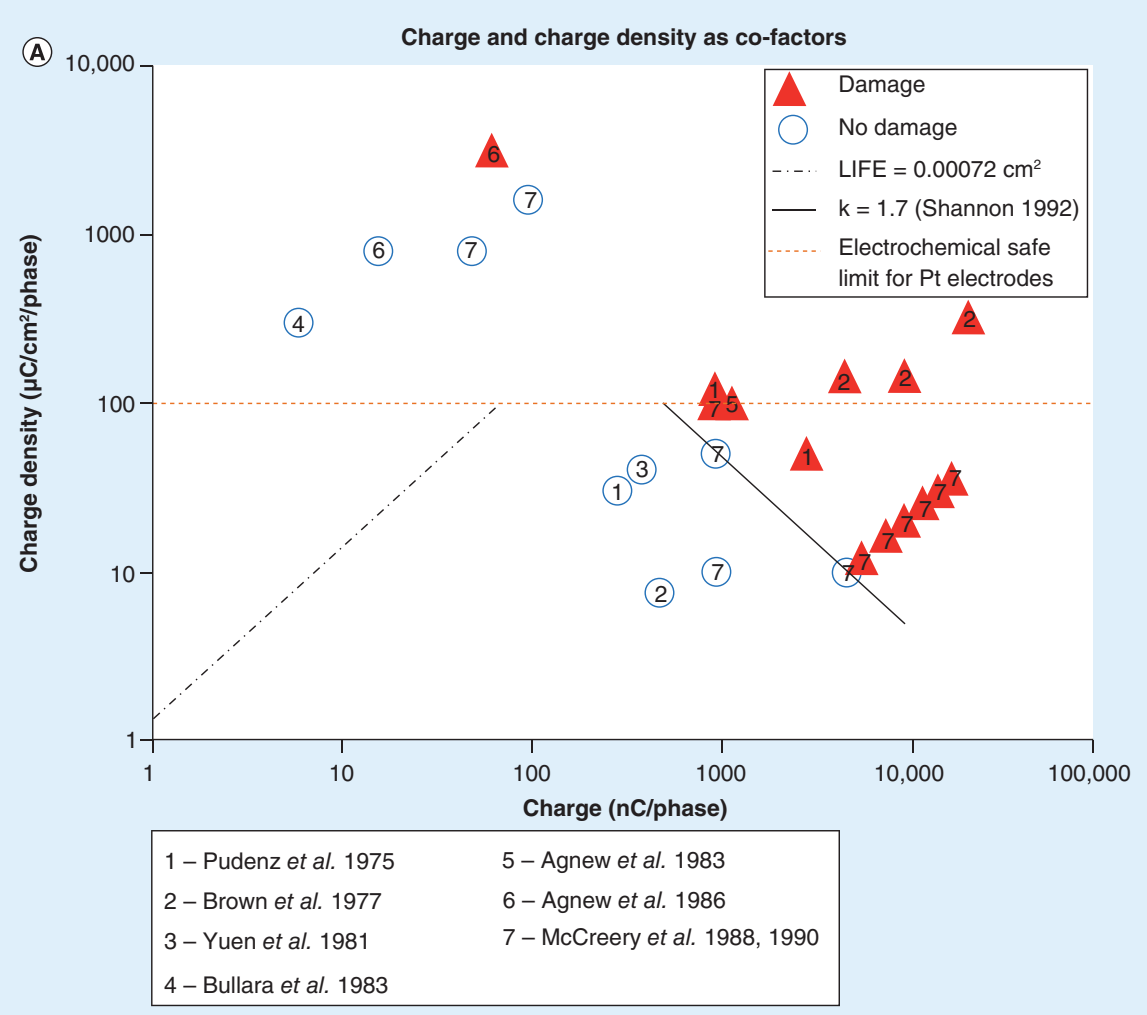

B Charge and charge density as co-factors

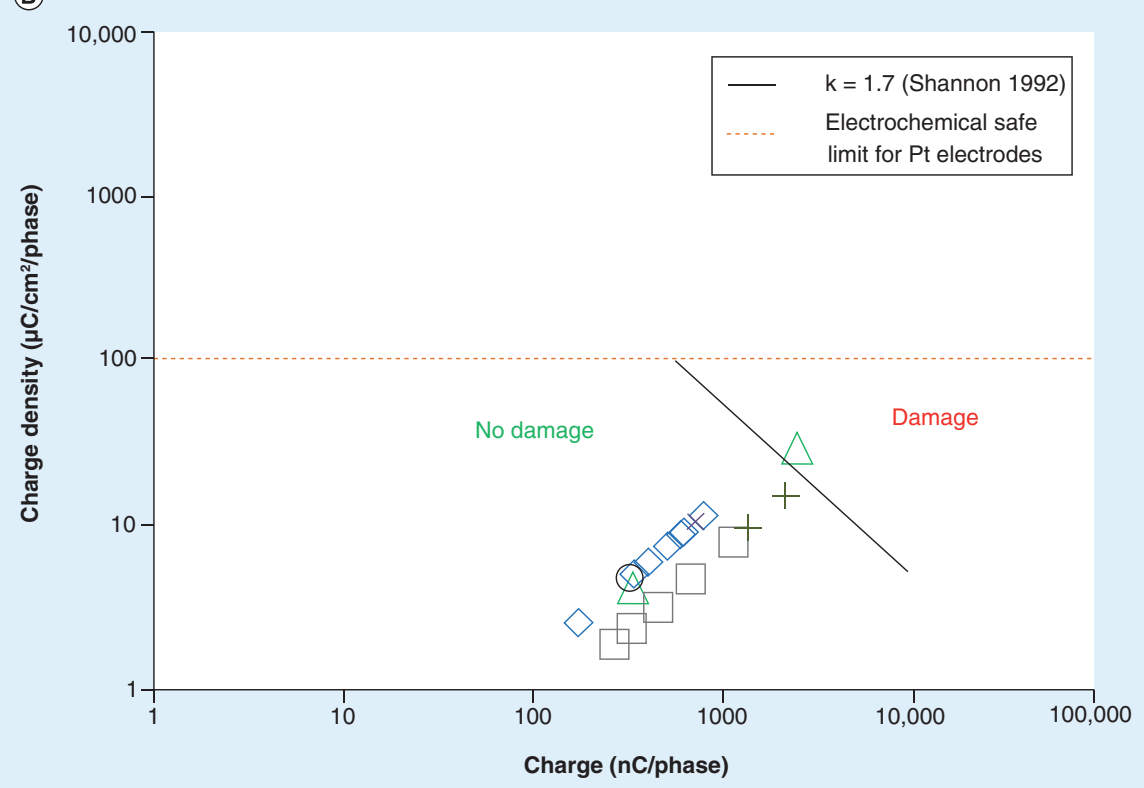

\begin{tabular}{|llll|}
\hline & Haberler et al 2002: DBS & $\bigcirc$ & Henderson et al 2002: DBS \\
$\square$ & Hanajima et al 2002: MC & + & Abejon et al 2007: SCS \\
$\triangle$ & Kinoshita et al 2004: EPI & $\times$ & Burbaud et al 2002: DBS \\
\hline
\end{tabular}

Figure 1. Neural injury in preclinical and clinical studies as a function of charge and charge density for macro electrodes.

(A) Data from several studies that delivered chronic stimulation via macro electrodes implanted in cortex in preclinical animal models [57-64]. Data points from animals in which damage was observed are indicated with red triangles; data points with no damage are indicated in blue circles; the number in each marker indicates the source of the data point. The limit for electrochemical safety is indicated by the orange dashed line and the region characterized as safe using the Shannon criterion is below and to the left of the solid black line. (B) Data points from electrodes implanted for stimulation of CNS structures in humans are presented in this plot [65-67,80-82].

DBS: Deep-brain stimulation; EPI: Epilepsy; MC: Motor cortex; SCS: Spinal cord stimulation. 


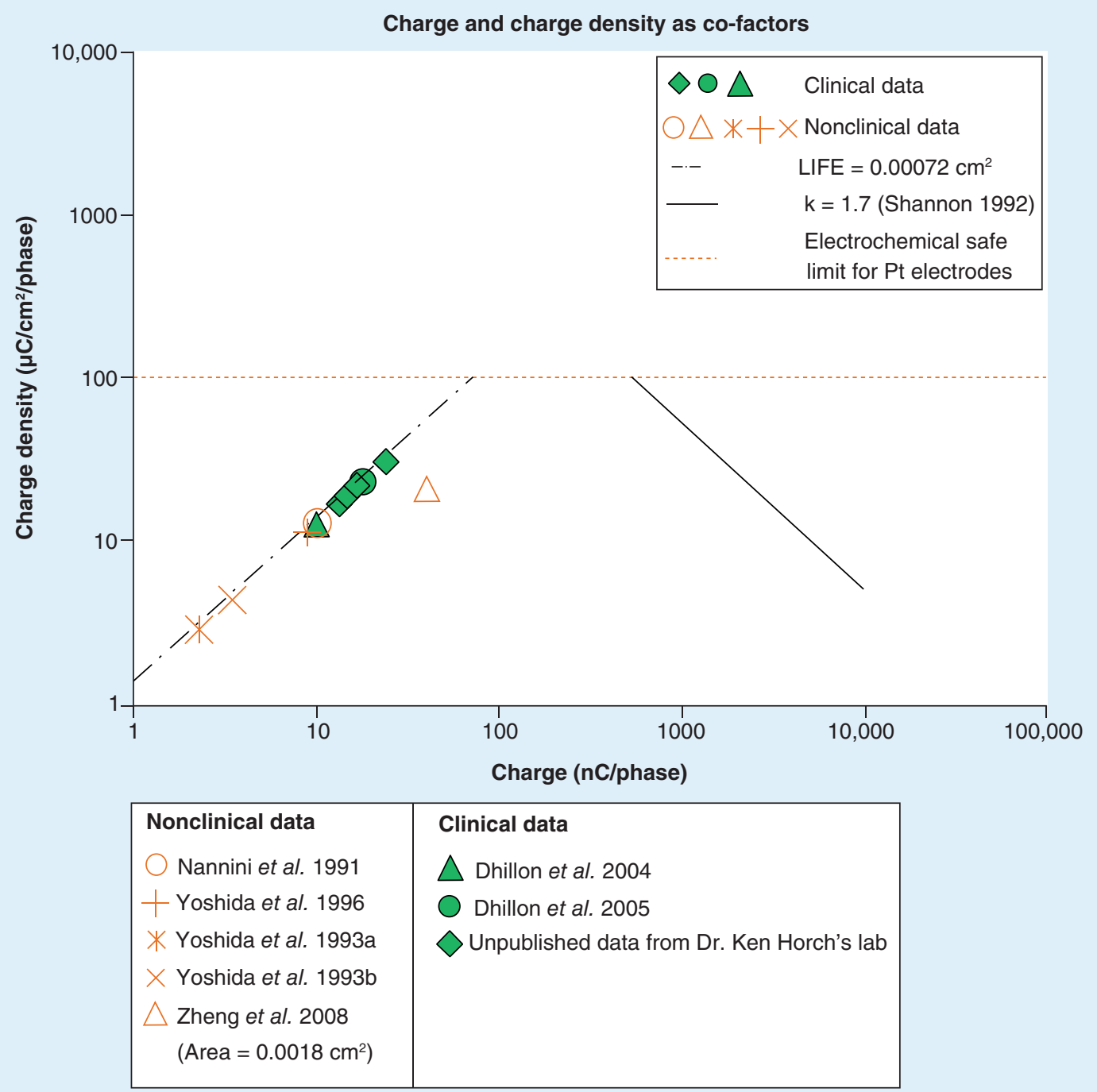

Figure 2. Charge and charge density parameters used in nonclinical and clinical studies with longitudinal intrafascicular electrodes.

These plots present data from a number of studies that delivered chronic stimulation via longitudinal intrafascicular electrodes implanted in peripheral nerves of chronic animal preparations (nonclinical data) and in human studies (clinical data) with durations of approximately 1 month. Neural damage was not observed in any of the studies and the stimulation parameters are well below the limit for electrochemical safety (below the orange dashed line) and within the region characterized as safe using the Shannon criterion (below and to the left of the solid black line) $[45,46,71-75]$.

Unpublished data provided courtesy of Dr. Ken Horch.

stimulation [75,76] and for ankle joint control [77]. Zheng et al. implanted a slightly modified version of LIFEs (diameter $=60 \mu \mathrm{m})$ in sciatic nerves of rabbits for a period of 6 months to study the recording and stimulating properties and biocompatibility of LIFEs [78]. In clinical studies (solid symbols in Figure 2), Dhillon et al. implanted Teflon-coated $25 \mu \mathrm{m}$ LIFEs in 15 human amputee subjects for periods ranging from 2 to 23 days [47,48]. In all of these studies the average stimulation levels tested was $<30 \mu \mathrm{C} / \mathrm{cm}^{2}$ which is well within levels considered to be safe for platinum macro electrodes [71].

\section{Intrafascicular electrodes: recording \& chronic biocompatibility}

A number of studies have investigated the recording properties and biocompatibility of Pt-Ir LIFEs in chronic animal studies for the duration of several months. Malagodi et al. [32] developed the $90 \%$ platinum-10\% iridium 
(Pt-Ir) LIFEs for implantation and recording in the fascicles of mammalian peripheral nerves. Pt-Ir LIFEs of $50 \mu \mathrm{m}$ diameter with a $1-2 \mathrm{~mm}$ active area could be used to record intrafascicularly from peripheral nerves. Using this length $(1-2 \mathrm{~mm})$ of exposed wire for the recording area maximized the signal-to-noise ratio while minimizing electrode impedance. Recordings remained stable for $48 \mathrm{~h}$ following implantation in cat ulnar nerve and no histological signs of inflammation, infection, extensive mechanical trauma, neuronal degeneration or myelin degeneration were found. Goodall et al. [45] implanted the same type of Pt-Ir LIFEs into the radial nerve of cats and recorded activity that was associated with discrete, nonoverlapping receptive fields. Over a period of 6 months, there was no change in the receptive fields for each electrode. Each electrode recorded activity from about ten afferent fibers, but the population of afferent fibers from which activity was recorded changed over time. While minor electrode migration occurred, such that the individual axons that made contact with the uninsulated electrode changed over time, the electrode did not migrate out of the fascicle or out of the nerve. Further, the total number of axons that could be recorded from one electrode at any given time did not change over the 6-month period, suggesting that the axons, fascicles and nerve were not damaged and that migration out of the nerve fascicle did not occur.

Lefurge et al. recorded from Pt-Ir LIFEs in the radial nerves of cats for up to 6 months and conducted histological analysis of fascicles from radial nerves with and without implants [31]. Each electrode could record from up to an average of ten axons during the 6 months. This finding corroborates the findings from Goodall et al. [45], suggesting that the axons and fascicles were not damaged, as they remained functionally stable. At the end of the 6-month testing period, sections from fascicles that had been implanted and those that had not been implanted were analyzed for: median diameter of myelinated axons at the site of implantation, and $1.0-1.5 \mathrm{~cm}$ proximal and $1.0-1.5 \mathrm{~cm}$ distal to the implant site; number of A $\alpha$ fibers near the uninsulated electrode; foreign body reactive changes at the recording site; and foreign body reactive changes at the endoneurium. The investigators found that at the implant site, there was a $40 \%$ reduction in axon diameter, which seemed to be the result of a decrease in myelination. However, at the sites proximal and distal to the implant site, there was no difference in axon diameter between implanted and control tissue. Further, ten or more $A \alpha$ fibers were found near the recording site for each electrode, even when electrodes could not record from all ten fibers. This finding suggests that axons around the electrode were not damaged, but that the electrical properties of the connective tissue in the area may have changed over time. Indeed, there appeared to be some minor reactive changes in the tissue surrounding the implant, with the appearance of a thin layer of multinucleate giant cells that were not actively phagocytosing, and alternating layers of collagen and fibroblasts with a total thickness of $<50 \mu \mathrm{m}$. An inflammatory reaction was not evident as granular leukocytes were not observed. In addition, there was an increase in endoneurial connective tissue around the implant site and a reduction in myelinated axons [31].

Zheng et al. [78] implanted a slightly different version of longitudinal intrafascicular electrodes (LIFE-2-60 $\mu \mathrm{m}$ $\mathrm{Pt}(95 \%) / \mathrm{Ir}(5 \%)$ wire) in the sciatic nerve fascicles of rabbits for a period of 6 months. Over this period, they used LIFEs to record motor-evoked potentials while stimulating the electrodes. They also recorded cortical somatosensory-evoked potentials while using LIFEs as stimulating electrodes. At the end of 6 months, the fascicles implanted with electrodes were histologically examined under light and transmission electron microscopes. They found that the onset latency of motor-evoked potentials and cortical somatosensory-evoked potentials had no obvious change during the first month, but the onset latency increased during the second month and then stabilized after 3 months. The fascicular tissue showed mild damage on histological analysis. No inflammatory cells or neuromas were observed around the electrode. The results from this study support the safety of chronic stimulation using LIFEs implanted in fascicles as long as the stimulation parameters are well within the safety limits imposed by the charge injection limits of the electrode material.

In an effort to compare biocompatibility of polyimide-based tf-LIFEs and Pt/Ir-LIFEs, Lago et al. [79] chronically implanted both types of LIFEs in the rat sciatic nerve for a period of 3 months. The LIFEs were made from Tefloninsulated $\mathrm{Pt}(90 \%) / \mathrm{Ir}(10 \%)$ wire with a bare wire diameter of $25 \mu \mathrm{m}$ and an effective insulated diameter of 60-75 $\mu \mathrm{m}$. However, instead of implanting a single wire, they formed a dual channel LIFE by bending a single wire into two halves and inserted this as one electrode. Hence, the effective diameter of the implanted electrode was approximately $120-150 \mu \mathrm{m}$, which is significantly larger than that used in the previous studies. Electrophysiological, functional tests and terminal morphological analysis were conducted in order to assess changes to the nerve as a result of electrode implantation. Nerve conduction studies compared the implanted nerve with the intact contralateral nerve. Compound muscle action potentials and compound nerve action potentials were also recorded from both sides to assess the functional properties of the nerves and innervation of distal targets. Functional evaluation 
studies included algesiometry test, walking track test and pinprick test [79]. The electrophysiological and functional measurements were made at 30,60 and 90 days postimplantation. At the end of 3 months, the animal was fixed and perfused. Subsequently, the sciatic nerve was extracted and dissected and processed for light microscopy and immunochemistry. The results indicated that compared with the intact nerve, the latencies of sensory and motor nerve fibers were slightly longer at 30 days post implant but returned to normal values when recorded at 60 and 90 days post implant. The amplitudes of compound muscle action potentials and compound nerve action potentials reduced significantly at 30 days and then increased toward normal levels at 60 and 90 days. Plantar algesiometry tests yielded similar values for hot and pain threshold for withdrawal. There was no evidence of hyperalgesia that might be induced by compression or nerve injury, no loss of sensitivity in any area of the hind paw under the pinprick test and walking and no abnormalities in locomotion or differences between the right and left hind limbs at any of the post implant times. Morphological tests showed that Pt/Ir-LIFEs induced a mild scar response and a focal but chronic inflammatory reaction which was limited to a very small area around the nerve. The studies by Lefurge et al. [31], and Zheng et al. [78] did not find any indication of inflammation. This difference could be because of the significantly larger cross-sectional area and hence stiffer electrodes used by Lago et al. [79]. In general, the results showed that Pt/Ir-LIFEs and tf-LIFEs are biocompatible and the functional loss seen at the end of 30 days was slight and reversible.

Taken together, these results indicate that axons within implanted fascicles remain functional over 6 months, and although there was some buildup of connective tissue around the implant (as with any other implant), there was minimal damage to the tissue as indicated by histological analysis of myelin loss, axonal (focal, nonfocal, Wallerian type) degeneration and traumatic fibrosis of peri/epineurium [31,78,79], and no evidence of an inflammatory response as indicated by the number of polymorphonuclear, lymphocytes and macrophages $[31,78,79]$. The fact that stable neural recordings were obtained indicates the tissue and the nerve-tissue interface remained functional.

\section{Implementation considerations: lead wires \& tethering forces}

A complete bionic interface system consists of multiple components, the electrode that forms the neural-tissue interface, the electronics for recording or stimulation and connecting leads. The lead system must have the strength and durability to withstand mechanical stresses and strains during surgical implantation and throughout years of chronic use. Additionally, since peripheral nerves can slide and stretch, movements may impact the stability of the electrode-nerve interface. Bench testing of the lead system can provide insight into potential failure mechanisms and strengthen the case for long-term viability in eventual human use. These tests should be designed to mimic the stresses anticipated in clinical use, which may include stresses induced during implantation and stresses imposed during daily activities. For intrafascicular electrodes it is especially important to assure that tethering forces do not damage the lead or the interface. Pena et al. have recently described the assessment of a lead system for deployment of a branched lead with LIFEs for implantation in the upper arm of amputees [56].

\section{Implementation considerations: bidirectional communication \& power}

Many designs of neural interface systems require communication between the implant and external components. The communication may be required intermittently for system configuration (e.g., DBS [80], spinal cord stimulation [81]) and/or continuously for system operation and power transmission (e.g., cochlear implants [82], Freehand system [83]). The use of wireless transmission of power to the implant and uni- or bidirectional communication of data can avoid the risks associated with percutaneous leads, but increases system complexity and may greatly increase power demands.

Neural implants are usually powered either via an implantable battery or through wireless power transfer from an external power source. The choice of power source employed is usually dictated by the application. Allowable implant size, implant power requirements and tolerance to power interruptions are some critical factors to be considered while deciding the type of power source for a given application [84]. Battery-powered neural implants enable the development of a truly implantable closed loop systems with no external components. However, they may require additional surgeries for battery replacement or a mechanism to transcutaneously recharge the battery [84]. Systems that use wireless power transfer utilize an external power source and a link (usually inductive) to transmit power to the implant. Such systems overcome the need for repeated surgeries for battery replacement [84]. The inductive wireless link used for power transfer can also be used for bidirectional transmission of information between the implanted unit and external processors. Some key factors affecting the design of wireless data transfer links are channel bandwidth and transmission range [2]. Intrafascicular electrodes are in close proximity to neural tissue 
Table 1. Bionic neural interfaces: summary of trade-offs, conclusion and future trends.

\begin{tabular}{|c|c|}
\hline Advantages: & $\begin{array}{l}\text { - Noninvasive to the nerve } \\
\text { - Comparatively simple to implant } \\
\text { - Proven long-term safety and stability }\end{array}$ \\
\hline Disadvantages: & $\begin{array}{l}\text { - For stimulation, intrafascicular selectivity may be a limiting factor for some applications } \\
\text { - For recording, selectivity is poor } \\
\text { - Suitability for use on small nerves is uncertain } \\
\text { - Simplicity of implantation may be limited if distributed sites are targeted }\end{array}$ \\
\hline Conclusion: & May be best suited for applications that seek to stimulate different fascicles within a large nerve bundle \\
\hline Future trends: & Increased selectivity, designs for smaller nerves, integration with intrafascicular designs \\
\hline \multicolumn{2}{|c|}{ Multicontact intrafascicular electrode arrays (rigid and flexible designs, e.g., USEA, tfLIFE, TIME) } \\
\hline Advantages: & $\begin{array}{l}\text { - Potential for high selectivity } \\
\text { - Selective access to multiple sets of nerve fibers within a fascicle with a single insertion } \\
\text { - Ability to access fibers deep in a fascicle }\end{array}$ \\
\hline Disadvantages: & $\begin{array}{l}\text { - Challenging to place into a fascicle (if going into a multifascicular nerve without exposing fascicles); } \\
\text { challenging to place within the fascicle to obtain multichannel functionality } \\
\text { - Stability is uncertain: electrode/substrate degradation, movement, encapsulation (especially for rigid } \\
\text { arrays) } \\
\text { - Potential for nerve damage is comparatively high, especially for rigid arrays } \\
\text { - Yield on implanted electrodes may be low }\end{array}$ \\
\hline Conclusion: & $\begin{array}{l}\text { May be best suited for sites where array can be securely anchored to the nerve bundle to minimize } \\
\text { potential for damage or instability of the interface }\end{array}$ \\
\hline Future trends: & Increased longevity and stability, improved targeting, enhanced flexibility \\
\hline \multicolumn{2}{|c|}{ Sets of individual LIFEs } \\
\hline Advantages: & $\begin{array}{l}\text { - High selectivity for stimulation and recording } \\
\text { - Ability to access fibers deep in a fascicle } \\
\text { - May provide good access to small fibers within a fascicle } \\
\text { - Proven long-term safety and stability } \\
\text { - Potentially suitable for accessing distributed sites across small nerve bundles }\end{array}$ \\
\hline Disadvantages: & $\begin{array}{l}\text { - Surgical implantation is comparatively time-consuming and challenging } \\
\text { - Inability to precisely target specific intrafascicular structures } \\
\text { - Yield on implanted electrodes may be low }\end{array}$ \\
\hline Future trends: & Simplified implantation, improved targeting and enhanced flexibility \\
\hline
\end{tabular}

and hence require low levels of charge injection to activate surrounding tissue in stimulation applications [2]. This in turn reduces the power requirements for the neural stimulator. For multichannel systems, the bandwidth and power requirements will scale with channel count and therefore may push the limits of currently available battery and communication capabilities.

\section{Conclusion}

The requirements for safety and stability of a bionic neural interface are paramount and are generally consistent across applications. For any type of interface, the primary demand for safety includes the requirement to avoid mechanical, electrical or chemical damage during implantation, long-term use, and explantation. For long-term use, the functionality of the interface requires stability, which implies avoiding substantial movement of the electrode with respect to the target tissue and avoiding substantial changes in the electrical properties of the interface.

For a given application, the selection of a suitable bionic interface will depend strongly on the specific anatomy of the targeted site and the physiology of the intended application. Specific anatomical constraints may be derived from the size of the nerve bundle, fascicular organization, fiber size distribution and the number and distribution of target sites. Specific physiological needs and constraints may place high demands on selectivity and on the ability to coordinate stimulation and/or recording across multiple sites. Application-specific safety concerns, such as the need to avoid inadvertent stimulation of nearby sites, may increase the demands for selectivity and stability.

Table 1 provides a summary of our views on the advantages and disadvantages of three general categories of bionic interface designs. For electrodes that are placed inside the nerve fascicle, one of the primary characteristics is the number of electrodes per insertion. LIFEs have been designed with one electrode per wire; several other 
designs have been fabricated so that each insertion includes multiple recording/stimulation sites (e.g., USEA, TIME, tfLIFE). The summary in Table 1 uses this very general categorization of single-wire versus multielectrode array to contrast the primary advantages and disadvantages of the two approaches. We have included a category for multicontact cuffs in this summary because they have undergone substantial use in clinical applications and, for many applications in bioelectronic medicine, they may be a viable alternative to intrafascicular designs.

Table 1 also includes statements of our general 'conclusion' regarding each interface category. These are based on our understanding of the benefits and limitations of each category and of the types of challenges faced for various applications. These conclusions, as well as the summaries of advantages and disadvantages, are based on information from the literature as much as possible, but they also admittedly rely to some degree on personal experience and on speculation. In our opinion, multicontact cuffs may be best suited for applications that seek to stimulate different fascicles within a large nerve bundle. This approach allows a single deployment of an array to address several fascicles; the degree of intrafascicular selectivity that may be achieved with a multicontact cuff is likely to be lower than that of intrafascicular designs. Multielectrode intrafascicular arrays may be best suited for sites where the array can be securely anchored to the nerve bundle to minimize the potential for damage or instability of the interface. The multielectrode array will achieve high channel counts and secure anchoring may be able to minimize movement between an individual electrode and the target tissue when under mechanical stress. LIFEs may be best suited for applications that demand high intrafascicular selectivity with a moderate number of channels. This approach can provide high selectivity and good stability, but the inability to target specific intrafascicular structures and the fact that an insertion is required for each electrode present substantial challenges when a large number of channels are required.

\section{Future perspective}

Neural engineering has emerged as a paradigm that links living with nonliving systems to investigate, intervene and harness neural plasticity to counter disease and disability. Fueled by engineering breakthroughs, the last two decades have seen a phenomenal rise in our ability to probe the nervous system and to influence neural system activity across scales of complexity and states of disease. Newly developed and emerging methods for precise fabrication at the nanoscale promise to greatly improve our ability to communicate with the nervous system at the cellular level. Neuromorphic electronic design in silicon or other organic computing platforms would allow realization of computational neural models by fabrication of fundamental devices and circuits that can emulate neuronal behavior at the single cell level as well as more complex circuits. These new biomimetic devices offer new platforms for bidirectional bionic neural interfaces [85].

Many advances in the future will be achieved by enhancing existing technologies. Improvements will address current limitations to enhance performance and to enable implementation for a wider range of applications. The next generations of multicontact cuffs may achieve greater intrafascicular selectivity by developing techniques to place electrodes closer to the fascicle, by creating new stimulation strategies or by integrating intrafascicular components into the cuff design. The next generations of multichannel intrafascicular arrays may achieve longer term stability and better placement accuracy by utilizing alternative materials for long-term survival and improved match with tissue mechanical properties, and by developing insertion techniques to improve placement within a fascicle. The next generations of LIFEs may achieve higher yields and more widespread adoption by developing tools and implantation techniques that allow placement near targeted intrafascicular structures, streamline surgical procedures, and minimize the need for specialized surgical training.

Other advances will be based on technologies that are currently being developed or are yet to be conceived. Improved electrical interfaces may be produced by utilizing newly developed materials in electrodes, electrode coatings or conductors to improve flexibility, reduce size and increase the capacity to focally stimulate or to record. While most peripheral neural activation has utilized electrical stimulation, magnetic, optical or chemical signals may also prove to be practical for focal stimulation or recording from peripheral nerve microdomains. Recently, novel technology using magnetoelectric nanoparticles for noninvasive focal neural stimulation using low-intensity direct current (DC) and alternating current (AC) magnetic fields has been developed [86]. In the CNS, optogenetic techniques are being utilized to allow activation of neural cells. For peripheral nerves, infrared neural stimulation techniques using lasers could evoke action potentials by providing a transient, targeted optical input that allows a very high degree of spatial resolution since only tissue in the path of the laser beam is activated. Chemical sensors or localized delivery of neurotransmitters or neuromodulators may be used to record, initiate or modulate neural activity. These techniques, which could be used either alone or in conjunction with electrical interfaces, could result 
in systems with improved selectivity and, perhaps more importantly for applications in bioelectronic medicine, improved ability to modulate endogenous neural activity.

\section{Executive summary}

Background

- Peripheral nerves provide gateways for modulation of the CNS and internal organs.

- Bionic interfaces can be used to induce patterns of activation or record neural activity.

- Cuff-like structures wrapped around nerves enable reliable stimulation, but some clinical applications may require high intrafascicular selectivity.

- Bionic intrafascicular interfaces may enable enhanced stimulation selectivity and provide the ability to record from small groups of fibers within a fascicle.

Intrafascicular electrodes: stimulation

- Several intrafascicular electrodes have been designed and utilized in preclinical and human studies: the Utah slant electrode array, longitudinal intrafascicular electrodes (LIFEs), thin-film LIFEs and the transverse intrafascicular multichannel electrode array.

Intrafascicular electrodes: choosing stimulation parameters

- The proximity of intrafascicular electrodes to target tissues enables low charge delivery.

- Electrodes are classified as 'macro' or 'micro' depending on their surface area.

- The safety of chronic stimulation using macro electrodes in brain structures has been investigated in several preclinical and clinical studies.

- The safety of chronic stimulation using LIFEs in peripheral nerves has been studied in long-term preclinical studies and in human studies with a duration of less than 1 month.

Intrafascicular electrodes: recording \& chronic biocompatibility

- The recording properties and biocompatibility of LIFEs and thin-film LIFEs have been investigated in several preclinical studies.

- Histological analysis indicates little or no signs of damage or inflammatory response.

- The stability of recording capability of LIFEs over several months is an indicator of long-term viability of the electrode, the surrounding tissue and the tissue-electrode interface.

Implementation considerations: lead wires \& tethering forces

- Electrode leads, which connect intrafascicular and cuff electrodes to implanted or external electronics, must be durable and must not induce damage to the nerve.

- Bench testing of the leads that mimics stresses of implantation and daily use can provide insight into potential failure mechanisms and strengthen the case for long-term viability in eventual human use.

Implementation considerations: power \& bidirectional interfaces

- Communication with the implanted and external components may be required intermittently during system configuration and/or continuously during system operation.

- Power requirements (for implanted electronics, stimulation and communication) may be met through the use of implanted batteries or transcutaneous power transmission.

Conclusion

- For a given application, the selection of a suitable bionic interface will depend strongly on the specific anatomy of the targeted site and the physiology of the intended application.

- The comparative advantages and disadvantages of multicontact cuffs, multicontact intrafascicular arrays and sets of LIFEs are outlined.

Future perspective

- Advances in bionic neural interfaces will be achieved by improvements to existing electrode designs and by exploiting new approaches to activating and monitoring neural activity.

Financial \& competing interests disclosure

This work was funded by the NIH through a National Institute of Biomedical Imaging and Bioengineering and a National Institute of Child Health and Human Development grant NIH-R01-EB008578. The authors have no other relevant affiliations or financial involvement with any organization or entity with a financial interest in or financial conflict with the subject matter or materials discussed in the manuscript apart from those disclosed.

No writing assistance was utilized in the production of this manuscript. 


\section{References}

Papers of special note have been highlighted as: $\bullet$ of interest; $\bullet \bullet$ of considerable interest.

1 Jung R. Merging technology with biology. In: Biohybrid Systems: Nerves, Interfaces, and Machines. Jung R (Ed.). Wiley-VCH, Weinheim, Germany, 213 (2011).

2 Horch KW. Peripheral nervous system. In: Neuroprosthetics Theory and Practice. Horch KW, Kipke DR (Eds). World Scientific River Edge, NJ, USA, 3-11 (2017).

-. Comprehensive book on neuroprosthetics, which covers topics in scientific fundamentals, electrode designs and clinical applications.

3 Matthew S, Daniel T, Steven MS, Dustin JT. Sensory feedback by peripheral nerve stimulation improves task performance in individuals with upper limb loss using a myoelectric prosthesis. J. Neural. Eng. 13(1), 016001 (2016).

4 Tan DW, Schiefer MA, Keith MW, Anderson JR, Tyler J, Tyler DJ. A neural interface provides long-term stable natural touch perception. Sci. Transl. Med. 6(257), 257ra138-257ra138 (2014).

- Clinical application of multicontact cuffs to restore sensation to human amputees that demonstrates fascicular selectivity and stability of the interface.

5 Christie BP, Freeberg M, Memberg WD et al. Long-term stability of stimulating spiral nerve cuff electrodes on human peripheral nerves. J. Neuroeng. Rehabil. 14(1), 70 (2017).

6 Gonzalez-Bermejo J, Llontop C, Similowski T, Morélot-Panzini C. Respiratory neuromodulation in patients with neurological pathologies: for whom and how? Annal. Phys. Rehab. Med. 58(4), 238-244 (2015).

7 Parhizgar F, Nugent K, Raj R. Obstructive sleep apnea and respiratory complications associated with vagus nerve stimulators. J. Clin. Sleep Med. 7(4), 401-407 (2011).

8 Koopman FA, Chavan SS, Miljko S et al. Vagus nerve stimulation inhibits cytokine production and attenuates disease severity in rheumatoid arthritis. Proc. Natl Acad. Sci. USA 113(29), 8284-8289 (2016).

9 Engineer ND, Riley JR, Seale JD et al. Reversing pathological neural activity using targeted plasticity. Nature 470(7332), 101-104 (2011).

10 Famm K, Litt B, Tracey KJ, Boyden ES, Slaoui M. Drug discovery: a jump-start for electroceuticals. Nature 496(7444), 159-161 (2013).

11 Katona PG. Biomedical engineering in heart-brain medicine: a review. Cleve. Clin. J. Med. 77(Suppl 3) S46-50 (2010).

12 Cohen ML, Georgievskaya Z. Histopathology of the stimulated vagus nerve: primum non nocere. Heart Fail. Rev. 16(2), 163-169 (2011).

13 Tyler DJ, Durand DM. Functionally selective peripheral nerve stimulation with a flat interface nerve electrode. IEEE Trans. Neural Syst. Rehabil. Eng. 10(4), 294-303 (2002).

14 Sweeney JD, Crawford NR, Brandon TA. Neuromuscular stimulation selectivity of multiple-contact nerve cuff electrode arrays. Med. Biol. Eng. Comput. 33(3 Spec No), 418-425 (1995).

15 Plachta DT, Gierthmuehlen $\mathrm{M}$, Cota $\mathrm{O}$ et al. Blood pressure control with selective vagal nerve stimulation and minimal side effects. J. Neural. Eng. 11(3), 036011 (2014).

16 Badia J, Boretius T, Andreu D, Azevedo-Coste C, Stieglitz T, Navarro X. Comparative analysis of transverse intrafascicular multichannel, longitudinal intrafascicular and multipolar cuff electrodes for the selective stimulation of nerve fascicles. J. Neural. Eng. 8(3), 036023 (2011).

- Compares versions of multicontact intrafascicular electrodes with multicontact cuffs.

17 Hallin RG. Microneurography in relation to intraneural topography: somatotopic organisation of median nerve fascicles in humans. J. Neurol. Neurosurg. Psychiatry 53(9), 736-744 (1990).

18 Stewart JD. Peripheral nerve fascicles: anatomy and clinical relevance. Muscle Nerve 28(5), 525-541 (2003).

19 Sun K, Zhang J, Chen T et al. Three-dimensional reconstruction and visualization of the median nerve from serial tissue sections. Microsurgery 29(7), 573-577 (2009).

20 Verlinden TJ, Rijkers K, Hoogland G, Herrler A. Morphology of the human cervical vagus nerve: implications for vagus nerve stimulation treatment. Acta Neurol. Scand. 133(3), 173-182 (2016).

21 Hallin RG, Wu G. Fitting pieces in the peripheral nerve puzzle. Exp. Neurol. 172(2), 482-492 (2001).

22 Gustafson KJ, Grinberg Y, Joseph S, Triolo RJ. Human distal sciatic nerve fascicular anatomy: implications for ankle control using nerve-cuff electrodes. J. Rehabil. Res. Dev. 49(2), 309-321 (2012).

23 Gustafson KJ, Pinault GC, Neville JJ et al. Fascicular anatomy of human femoral nerve: implications for neural prostheses using nerve cuff electrodes. J. Rehabil. Res. Dev. 46(7), 973-984 (2009).

24 Venkatasubramanian G, Jung R, Sweeney JD. Functional electrical stimulation. In: Encyclopedia of Medical Devices and Instrumentation. Webster JG (Ed.). Wiley-Interscience Hoboken, NJ, USA, 347-366 (2006). 
25 Navarro X, Krueger TB, Lago N, Micera S, Stieglitz T, Dario P. A critical review of interfaces with the peripheral nervous system for the control of neuroprostheses and hybrid bionic systems. J. Peripher. Nerv. Syst. 10(3), 229-258 (2005).

26 Christopher RB, Ian OM, Richard AN, Gregory AC. Selective neural activation in a histologically derived model of peripheral nerve. J. Neural. Eng. 8(3), 036009 (2011).

27 Veltink PH, Van Alste JA, Boom HB. Multielectrode intrafascicular and extraneural stimulation. Med. Biol. Eng. Comput. 27(1), 19-24 (1989).

28 Grill WM Jr. Modeling the effects of electric fields on nerve fibers: influence of tissue electrical properties. IEEE Trans. Biomed. Eng. 46(8), 918-928 (1999).

29 Bourbeau DJ, Hokanson JA, Rubin JE, Weber DJ. A computational model for estimating recruitment of primary afferent fibers by intraneural stimulation in the dorsal root ganglia. J. Neural. Eng. 8(5), 056009 (2011).

30 Kent AR, Grill WM. Model-based analysis and design of nerve cuff electrodes for restoring bladder function by selective stimulation of the pudendal nerve. J. Neural. Eng. 10(3), 036010 (2013).

31 Lefurge T, Goodall E, Horch K, Stensaas L, Schoenberg A. Chronically implanted intrafascicular recording electrodes. Ann. Biomed. Eng. 19(2), 197-207 (1991).

32 Malagodi MS, Horch KW, Schoenberg AA. An intrafascicular electrode for recording of action-potentials in peripheral-nerves. Ann. Biomed. Eng. 17(4), 397-410 (1989).

33 Kundu A, Harreby KR, Yoshida K, Boretius T, Stieglitz T, Jensen W. Stimulation selectivity of the "thin-film longitudinal intrafascicular electrode" (tfLIFE) and the "transverse intrafascicular multi-channel electrode" (TIME) in the large nerve animal model. IEEE Trans. Neural Syst. Rehabil. Eng. 22(2), 400-410 (2014).

- Describes the use of thin-film longitudinal intrafascicular electrode (LIFE) and transverse intrafascicular multichannel electrode in animal model and illustrates potential advantages and disadvantages of the approaches.

34 Boretius T, Badia J, Pascual-Font A et al. A transverse intrafascicular multichannel electrode (TIME) to interface with the peripheral nerve. Biosens. Bioelectron. 26(1), 62-69 (2010).

35 Davis TS, Wark HA, Hutchinson DT et al. Restoring motor control and sensory feedback in people with upper extremity amputations using arrays of 96 microelectrodes implanted in the median and ulnar nerves. J. Neural. Eng. 13(3), 036001 (2016).

36 Normann RA, Fernandez E. Clinical applications of penetrating neural interfaces and Utah Electrode Array technologies. J. Neural. Eng. 13(6), 061003 (2016).

- Recent review of clinical applications of Utah electrode arrays.

37 Oddo CM, Raspopovic S, Artoni F et al. Intraneural stimulation elicits discrimination of textural features by artificial fingertip in intact and amputee humans. Elife 5, e09148 (2016).

38 Raspopovic S, Capogrosso M, Petrini FM et al. Restoring natural sensory feedback in real-time bidirectional hand prostheses. Sci. Transl. Med. 6(222), 222 ra219 (2014).

- Describes the transverse intrafascicular multi-channel electrode and its use in human subjects for stimulation and recording.

39 Rossini PM, Micera S, Benvenuto A et al. Double nerve intraneural interface implant on a human amputee for robotic hand control. Clin. Neurophysiol. 121(5), 777-783 (2010).

40 Horch K, Meek S, Taylor TG, Hutchinson DT. Object discrimination with an artificial hand using electrical stimulation of peripheral tactile and proprioceptive pathways with intrafascicular electrodes. IEEE Transactions on Neural Systems and Rehabilitation Engineering 19(5), 483-489 (2011).

- Describes the use of LIFEs in human amputee subjects and demonstrates the selectivity and high-quality percepts elicited by stimulation.

41 Branner A, Normann RA. A multielectrode array for intrafascicular recording and stimulation in sciatic nerve of cats. Brain Res. Bull. 51(4), 293-306 (2000).

42 Branner A, Stein RB, Fernandez E, Aoyagi Y, Normann RA. Long-term stimulation and recording with a penetrating microelectrode array in cat sciatic nerve. IEEE Trans. Biomed. Eng. 51(1), 146-157 (2004).

- Describes the Utah Slant Electrode Array and its use in peripheral nerves.

43 Wark HA, Sharma R, Mathews KS et al. A new high-density (25 electrodes/mm(2)) penetrating microelectrode array for recording and stimulating sub-millimeter neuroanatomical structures. J. Neural. Eng. 10(4), 045003 (2013).

44 Byun D, Cho SJ, Lee BH, Min J, Lee JH, Kim S. Recording nerve signals in canine sciatic nerves with a flexible penetrating microelectrode array. J. Neural. Eng. 14(4), 046023 (2017).

45 Goodall EV, Lefurge TM, Horch KW. Information contained in sensory nerve recordings made with intrafascicular electrodes. Biomedical Engineering, IEEE Transactions on 38(9), 846-850 (1991).

46 Lawrence SM, Dhillon GS, Jensen W, Yoshida K, Horch KW. Acute peripheral nerve recording characteristics of polymer-based longitudinal intrafascicular electrodes. IEEE Trans. Neural Syst. Rehabil. Eng. 12(3), 345-348 (2004). 
47 Dhillon GS, Lawrence SM, Hutchinson DT, Horch KW. Residual function in peripheral nerve stumps of amputees: implications for neural control of artificial limbs. The Journal of Hand Surgery 29(4), 605-615 (2004).

48 Dhillon GS, Horch KW. Direct neural sensory feedback and control of a prosthetic arm. IEEE Trans. Neural Systems Rehab. Eng. 13(4), 468-472 (2005).

49 Yoshida K, Stein RB. Characterization of signals and noise rejection with bipolar longitudinal intrafascicular electrodes. IEEE Trans. Biomed. Eng. 46(2), 226-234 (1999).

50 Thota AK, Kuntaegowdanahalli S, Starosciak AK et al. A system and method to interface with multiple groups of axons in several fascicles of peripheral nerves. J. Neurosci. Methods 244, 78-84 (2015).

- Describes a system developed for managing sets of LIFEs during surgical implantation.

51 Saal HP, Bensmaia SJ. Biomimetic approaches to bionic touch through a peripheral nerve interface. Neuropsychologia 79, 344-353 (2015).

52 Navarro X, Lago N, Vivo M et al. Neurobiological evaluation of thin-film longitudinal intrafascicular electrodes as a peripheral nerve interface. Presented at: 2007 IEEE 10th International Conference on Rehabilitation Robotics. Noordwijk, The Netherlands 13-15 Jun (2007). www.ieee.org/conferences_events/conferences/conferencedetails/index.html?Conf_ID=12386

53 Badia J, Boretius T, Pascual-Font A, Udina E, Stieglitz T, Navarro X. Biocompatibility of chronically implanted transverse intrafascicular multichannel electrode (TIME) in the rat sciatic nerve. IEEE Trans. Biomed. Eng. doi:10.1109/TBME.2011.2153850 (2011).

54 Lacour SP, Benmerah S, Tarte E et al. Flexible and stretchable micro-electrodes for in vitro and in vivo neural interfaces. Med. Biol. Eng. Comput. 48(10), 945-954 (2010).

55 Cutrone A, Del Valle J, Santos D et al. A three-dimensional self-opening intraneural peripheral interface (SELINE). J. Neural. Eng. 12(1), 016016 (2015).

56 Pena A, Kuntaegowdanahalli S, Abbas JJ, Patrick J, Horch KW, Jung R. Mechanical fatigue resistance of an implantable branched lead system for a distributed set of longitudinal intrafascicular electrodes. J. Neural Eng.14, 1-10 (2017).

57 Cogan SF. Neural stimulation and recording electrodes. Annu. Rev. Biomed. Eng. 10, 275-309 (2008).

-. Comprehensive presentation of key issues related to the design and use of electrodes for stimulation and recording as well as electrochemical safety concerns.

58 Cogan SF, Ehrlich J, Plante TD et al. Sputtered iridium oxide films for neural stimulation electrodes. J. Biomed. Mater. Res. B Appl. Biomater. 89(2), 353-361 (2009).

59 Shannon RV. A model of safe levels for electrical stimulation. IEEE Trans. Biomed. Eng. 39(4), 424-426 (1992).

60 Mccreery DB, Agnew WF, Yuen TG, Bullara L. Charge density and charge per phase as cofactors in neural injury induced by electrical stimulation. IEEE Trans. Biomed. Eng. 37(10), 996-1001 (1990).

61 Mccreery D, Agnew W, Yuen T, Bullara L. Comparison of neural damage induced by electrical stimulation with faradaic and capacitor electrodes. Ann. Biomed. Eng. 16(5), 463-481 (1988).

62 Yuen TG, Agnew WF, Bullara LA, Jacques S, Mccreery DB. Histological evaluation of neural damage from electrical stimulation: considerations for the selection of parameters for clinical application. Neurosurgery 9(3), 292-299 (1981)

63 Pudenz RH, Bullara LA, Jacques S, Hambrecht F. Electrical stimulation of the brain. III. The neural damage model. Surgical Neurology 4(4), 389-400 (1975).

64 Bullara L, Mccreery D, Yuen T, Agnew W. A microelectrode for delivery of defined charge densities. J. Neurosci. Methods 9(1), 15-21 (1983).

65 Agnew WF, Yuen TGH, Mccreery DB. Morphologic changes after prolonged electrical stimulation of the cat's cortex at defined charge densities. Experimental Neurology 79(2), 397-411 (1983).

66 Agnew W, Yuen T, Mccreery D, Bullara L. Histopathologic evaluation of prolonged intracortical electrical stimulation. Experimental Neurology 92(1), 162-185 (1986).

67 Brown WJ, Babb TL, Soper HV, Lieb JP, Ottino CA, Crandall PH. Tissue reactions to long-term electrical stimulation of the cerebellum in monkeys. J. Neurosurgery 47(3), 366-379 (1977).

68 Henderson JM, Pell M, O'sullivan DJ et al. Postmortem analysis of bilateral subthalamic electrode implants in Parkinson's disease. Movement Disorders 17(1), 133-137 (2002).

69 Haberler C, Alesch F, Mazal PR et al. No tissue damage by chronic deep-brain stimulation in Parkinson's disease. Annal. Neurol. 48(3), 372-376 (2000).

70 Burbaud P, Vital A, Rougier A et al. Minimal tissue damage after stimulation of the motor thalamus in a case of chorea-acanthocytosis. Neurology 59(12), 1982-1984 (2002).

71 Kuncel AM, Grill WM. Selection of stimulus parameters for deep-brain stimulation. Clin. Neurophysiol. 115(11), 2431-2441 (2004).

72 Green RA, Lovell NH, Wallace GG, Poole-Warren LA. Conducting polymers for neural interfaces: challenges in developing an effective long-term implant. Biomaterials 29(24-25), 3393-3399 (2008). 
73 Lotti F, Ranieri F, Vadala G, Zollo L, Di Pino G. Invasive intraneural interfaces: foreign body reaction issues. Front. Neurosci. 11, 497 (2017).

74 Nannini N, Horch K. Muscle recruitment with intrafascicular electrodes. IEEE Trans. Biomed. Eng. 38(8), 769-776 (1991).

75 Yoshida K, Horch K. Reduced fatigue in electrically stimulated muscle using dual channel intrafascicular electrodes with interleaved stimulation. Ann. Biomed. Eng. 21(6), 709-714 (1993).

76 Yoshida K, Horch K. Selective stimulation of peripheral nerve fibers using dual intrafascicular electrodes. IEEE Trans. Biomed. Eng. 40(5), 492-494 (1993).

77 Yoshida K, Horch K. Closed-loop control of ankle position using muscle afferent feedback with functional neuromuscular stimulation. IEEE Trans. Biomed. Eng. 43(2), 167-176 (1996).

78 Zheng X, Zhang J, Chen T, Chen Z. Recording and stimulating properties of chronically implanted longitudinal intrafascicular electrodes in peripheral fascicles in an animal model. Microsurgery 28(3), 203-209 (2008).

79 Lago N, Yoshida K, Koch KP, Navarro X. Assessment of biocompatibility of chronically implanted polyimide and platinum intrafascicular electrodes. IEEE Trans. Biomed. Eng. 54(2), 281-290 (2007).

80 Thevathasan W, Gregory R. Deep-brain stimulation for movement disorders. Practical Neurology 10(1), 16-26 (2010).

81 Wolter T. Spinal cord stimulation for neuropathic pain: current perspectives. J. Pain Res. 7, 651 (2014).

82 Zeng F-G, Rebscher S, Harrison W, Sun X, Feng H. Cochlear implants: system design, integration, and evaluation. IEEE Trans. Biomed. Eng. 1, 115-142 (2008).

83 Taylor P, Esnouf J, Hobby J. The functional impact of the freehand system on tetraplegic hand function. clinical results. Spinal Cord 40(11), 560 (2002).

84 Yilmaz G. Wireless Power Transfer. In: Wireless Power Transfer and Data Communication for Neural Implants. Case Study: Epilepsy Monitoring, Yilmaz G, Dehollain C (Eds). Springer International Publishing 23-55 (2017).

85 Jung R, Brauer EJ, Abbas JJ. Real-time interaction between a neuromorphic electronic circuit and the spinal cord. IEEE Trans. Neural Systems Rehab. Eng. 9(3), 319-326 (2001).

86 Guduru R, Liang P, Runowicz C, Nair M, Atluri V, Khizroev S. Magneto-electric nanoparticles to enable field-controlled high-specificity drug delivery to eradicate ovarian cancer cells. Sci. Rep. 3, 2953 (2013). 
